Copyright (C1996, American Institute of Aeronautics and Astronautics, Inc.

AIAA Meeting Papers on Disc, 1996, pp. 267-273

A9626827, AIAA Paper 96-1348

\title{
Acoustic vibration model for a composite shell with sound absorption material
}

\author{
Michael R. Dungan \\ Michigan Univ., Ann Arbor \\ William J. Anderson \\ Michigan Univ., Ann Arbor \\ Christopher Mollo \\ Automated Analysis Corp., Ann Arbor, MI \\ Nickolas Vlahopoulos \\ Automated Analysis Corp., Ann Arbor, MI
}

IN:AIAA/ASME/ASCE/AHS/ASC Structures, Structural Dynamics, and Materials Conference and Exhibit, 37th, Salt Lake City, UT, Apr. 15-17, 1996, Technical Papers. Pt. 1 (A96-26801 06-39), Reston, VA, American Institute of Aeronautics and Astronautics, 1996, p. 267-273

Reduction of sound transmitted through vehicle panels is increasingly important. A foam layer on vehicle panels is often used to passively control sound and small amplitude vibrations; this can be modeled and analyzed using the FEM only in 2D. This formulation assumes an isotropic structural member that couples with the elastic porous material. To expand beyond isotropic structures, fibrous composite plate and shallow shell elements are presented which were developed from the Mindlin plate assumptions extended to shear deformable and shallow shell composite laminates. The governing equations for the elastic porous material are extended to $3 \mathrm{D}$, and a procedure is described to convert these equations into a solid FEM formulation. The 2D foam-structure coupling leads to a determination of the coupling in the more general case. The development of this FEM formulation for elastic porous materials allows for designing low weight sound absorption systems with maximum transmission loss. (Author) 
$96-1348$

\title{
ACOUSTIC VIBRATION MODEL OF A COMPOSITE SHELL WITH SOUND ABSORPTION MATERIAL
}

\author{
Michael R. Dungan* \\ Dept. of Aerospace Engineering \\ University of Michigan \\ Ann Arbor, MI 48109-2118 \\ Christopher Mollo and Nickolas Vlahopoulos \\ Automated Analysis Corporation \\ 2805 South Industrial, Suite 100 \\ Ann Arbor, MI 48104-6767 \\ William J. Anderson $\uparrow$ \\ Dept. of Aerospace Engineering \\ University of Michigan \\ Ann Arbor, MI 48109-2118
}

\begin{abstract}
Reduction of sound transmitted through vehicle panels is increasingly important. A foam layer on vehicle panels is often used to passively control sound and small amplitude vibrations. Currently, this sound absorption layer can be modeled and analyzed using the finite element method only in two dimensions. This formulation assumes an isotropic structural member that couples with the elastic porous material. To expand beyond isotropic structures, fibrous composite plate and shallow shell elements are presented. These were developed from the Mindlin plate assumptions extended to shear deformable and shallow shell composite laminates. The governing equations for the elastic porous material are extended to three dimensions, and a procedure is described to convert these equations into a solid finite element formulation. The two-dimensional foam-structure coupling leads to a determination of the coupling in the more general case. The development of this finite element formulation for elastic porous materials allows for designing low weight sound absorption systems with maximum transmission loss.

\section{Introduction}

The object of study is vibration in plates and shells connected to an absorptive medium. Understanding these vibrations would allow for

* Graduate Student, Student Member AIAA.

Professor of Aerospace Engineering, Senior Member AIAA.

Copyright $\odot 1996$ by M.R. Dungan, C. Mollo, N. Vlahopolous, and

W.J. Anderson. Published by the American Institute of Aeronautics and Astronautics, Inc. with permission.
\end{abstract}

optimizing the structure for increased transmission loss in sound propagation. Current analysis for such a system is often completed using an analytic solution in one spatial dimension. ${ }^{1}$ For large flat plates and regular wall structures, this is a fairly good method for determining plane wave characteristics, such as reflection coefficient and transmission loss. However, for reliable analysis of structures with a more complex geometry, the finite element method is used.

Many sound absorptive materials resemble an elastic foam. Such a foam is composed of an elastic frame with fluid filled pores. It is the coupling between the vibration of such a fluid and the elastic frame that prevents the use of the typical structural dynamic finite element. Bolton, Shiau and Kang of Purdue University ${ }^{2}$ modified the theory of $\mathrm{Biot}^{3}$ to determine the equations that govern elastic wave propagation in elastic porous material. A two dimensional finite element of this foam has been developed by Kang, Bolton, Tsoi and Mollo. ${ }^{4}$

In two dimensions, the general problem is set up with three possible material regions: acoustic, elastic porous, and structural. The fluid region is modeled using finite elements based on the Helmholtz equation with nodal values being pressures. The interface conditions between the acoustic finite elements and those developed by Bolton, et al. are determined by energy methods to complete the relationship between applied harmonic forces and nodal displacements or pressures. $^{2}$

The vibrations of the structural region are modeled using an Euler-Bernoulli beam vibration formulation. This and the interface between the structural panel and the elastic porous material is to be covered in more detail. 
Fibrous composite laminates have an increasing role in aircraft and automotive applications. Thus it is important to model these structures as well. Acoustic energy is transmitted through these solid materials by vibration. For each element type, the energy formulation is converted into the familiar structural finite element equations of motion, assuming no damping and a harmonic time dependence. Shear deformation effects are included in the response of the composite laminate. The element derivation is from the Mindlin plate assumptions extended to shear deformable composite laminates composed of uniform orthotropic plies ${ }^{5,6}$. This derivation is covered in more detail as well.

The analysis of the response due to dynamic loading of the composite laminate/elastic porous system requires combining all of these finite element models in three dimensions. The plate elements of the composite laminate combine with the brick elements of the acoustic and elastic porous regions to successfully model the system behavior to acoustic excitation. A look at the basic procedure required to derive these elastic porous elements is presented.

\section{2-D Structural-Elastic Porous Formulation}

Bolton, Shiau and Kang of the Ray W. Herrick Laboratories at Purdue University ${ }^{2}$ developed the governing equations for wave propagation in elastic porous material. Based on the theory of Biot $^{3}$, these equations combine the dynamic equations of motion, assuming harmonic time dependence, and the stressstrain relations of both the solid and fluid phases of an elastic porous material:

$$
\begin{array}{r}
N \nabla^{2} u+\nabla\left[(A+N) e_{s}+Q \varepsilon\right]=-\omega^{2}\left(\rho_{11} u+\rho_{12} U\right)+i \omega b(u-U) \\
\nabla\left[Q e_{3}+R \varepsilon\right]=-\omega^{2}\left(\rho_{12} u+\rho_{22} U\right)-i \omega b(u-U)
\end{array}
$$

In these equations, $e_{s}=\nabla \bullet u=$ the solid volumetric strain, $\mathbf{u}=$ displacement vector for the structural phase, $\varepsilon=\nabla \bullet U=$ volumetric strain for the fluid phase, $\mathbf{U}=$ vector of fluid displacement, $\mathbf{N}=$ elastic shear modulus, and $\mathrm{A}=$ first Lame constant. The interaction between the volume change of the solid and elastic phases is quantified by coefficients $Q$ and R. $\rho_{11}, \rho_{12}$, and $\rho_{22}=$ mass coefficients depending on the porous tortuosity and representing the transferring of momentum between the solid and the fluid phase. The last term on the right hand side of both equations represents viscous coupling between the two phases. The two-dimensional finite element of this elastic porous material developed by Kang, Bolton, Tsoi and Mollo is based on these vector differential equations.

Combining the three material regions and their coupling interface results in this two-dimensional matrix form of equations:

$$
\left[\begin{array}{lll}
{\left[\mathrm{K}_{\mathrm{a}}\right]} & {\left[\mathrm{K}_{\mathrm{xf}}\right]} & {\left[\mathrm{K}_{\mathrm{w}}\right]} \\
{\left[\mathrm{K}_{\mathrm{ar}}\right]} & {\left[\mathrm{K}_{\mathrm{f}}\right]} & {\left[\mathrm{K}_{\mathrm{sf}}\right]} \\
{\left[\mathrm{K}_{\mathrm{w}}\right]} & {\left[\mathrm{K}_{\mathrm{sf}}\right]} & {\left[\mathrm{K}_{\mathrm{s}}\right]}
\end{array}\right]\left\{\begin{array}{c}
\{\mathrm{p}\} \\
\left\{\mathrm{u}_{\mathrm{x}}\right\} \\
\left\{\mathrm{u}_{\mathrm{y}}\right\} \\
\left\{\mathrm{U}_{\mathrm{x}}\right\} \\
\left\{\mathrm{U}_{\mathrm{y}}\right\} \\
\{\mathrm{W}\}
\end{array}\right\}=\left\{\begin{array}{c}
\{\mathrm{Q}\} \\
\left\{\mathrm{F}^{1}\right\} \\
\left\{\mathrm{F}^{2}\right\} \\
\left\{\mathrm{F}^{3}\right\} \\
\left\{\mathrm{F}^{4}\right\} \\
\{\mathrm{F}\}
\end{array}\right\}
$$

where $\left[K_{2}\right],\left[K_{2}\right]$, and $\left[K_{t}\right]$. are the acoustic stiffness and global dynamic stiffness matrix for the structure and elastic porous body, respectively. The other stiffness sub-matrices represent the coupling relations. Acoustic sound pressure is $p$, and $W$ is transverse displacement of the structural panel while $u_{x}, u_{y}, U_{x}$, and $U_{y}$ are components of displacement for the elastic porous material's solid and fluid phases, respectively The right hand side sub-vectors are force vectors: $[Q]$ is the normal volume velocity flux vector, $\{F\}$ is the external loads applied to the structure, and $\left\{F_{1}\right\}$ is related to the normal and shear stresses acting on the solid and fluid phases of the elastic porous body.

The structural finite elements are based on the bending vibration equation of motion for a onedimensional beam under pressure loading:

$$
\left(\frac{\mathrm{EI}}{1-v^{2}}\right) \frac{d^{4} \mathrm{w}_{t}}{d y^{4}}-\omega^{2} \mathrm{~m}_{\mathrm{p}} \mathrm{w}_{t}=p
$$

where $w_{1}$ is the transverse displacement, $m_{p}$ is the mass per unit area, $v$ is the Poisson's ratio and $I$ is the second moment of area per unit width.

Since the system dynamics of the panel are fully included in the formulation of the panel finite element, only the following two boundary condition are required at the elastic porous material/structural panel interface:

$$
\begin{aligned}
& u_{x}=w_{t} \\
& U_{x}=w_{t}
\end{aligned}
$$

The transverse displacement of the panel, $w_{t}$, matches the $x$-component displacements of the solid and fluid phases of the elastic porous material, $u_{x}$ and $U_{x}$, at the interface.

The interaction between the structural panel and the elastic porous body also depends on the strength of 
the connection. A numerical study was done to determine the effect of a panel being bonded to the elastic porous layer versus a panel loosely connected. A one-dimensional transmission loss test section was modeled. This section is set within two hard walls with a harmonic excitation at one end of the channel, and consists of an elastic porous layer (polyimide foam, $5.4 \mathrm{~cm}$ thick) sandwiched by two isotropic plates (aluminum, $0.762 \mathrm{~mm}$ thick), as shown in Figure 1 for the bonded panel case. The unbonded case is modeled by leaving a small gap of air $(0.5 \mathrm{~cm})$ between the panel and the foam layer as shown in Figure 2.

The results were computed by assuming no lateral constraints on the panels. Figure 3 does show that the two-dimensional finite element formulation closely matched the one dimensional analytical solution in determining transmission loss across the foam-panel system. The large error found at high frequencies is a result of the finite element mesh size being too large to accurate model the vibrations.

\section{Formulation of Composite Laminates}

Acoustic energy is transmitted through fibrous composite laminates by small amplitude vibrations. The traditional structural finite element equations of motion adequately model the dynamics of the composite plate for acoustics. Assuming no damping and a harmonic time dependence, this system of equations is written?:

$$
\left(-\omega^{2}[M]+[K]\right)\{u\}=\{F\}
$$

The structural degrees of freedom and the external loads applied to the structure are represented by $\{\mathrm{u}\}$ and $\{F\}$, respectively.

Classical laminate theory ${ }^{8}$ is used to compute the constitutive matrix of the laminate. This assumes that each layer of the laminate is orthotropic. After transforming the constitutive relation for each layer to an elemental coordinate system, these values are integrated through the thickness to yield the elemental constitutive relation:

$$
\left[\begin{array}{ll}
{[\mathrm{A}]} & {[\mathrm{B}]} \\
{[\mathrm{B}]} & {[\mathrm{D}]}
\end{array}\right]\left\{\begin{array}{c}
\left\{\varepsilon^{\mathrm{o}}\right\} \\
\{\kappa\}
\end{array}\right\}=\left\{\begin{array}{l}
\{\mathrm{N}\} \\
\{\mathrm{M}\}
\end{array}\right\}
$$

In-plane force resultants and resultant moments are represented by $N$ and $M$, respectively. The mid-plane strain vector is $\varepsilon^{\circ}$, and $\kappa$ is the mid-plane curvature vector. The $B$ sub-matrix clearly shows the coupling between in-plane forces and out-of-plane curvature that is not found in isotropic materials. The transverse shear constitutive relation was computed by integrating through the thickness while assuming each ply is transversely isotropic:

$$
\left[\begin{array}{ll}
\mathrm{A}_{44} & \mathrm{~A}_{45} \\
\mathrm{~A}_{45} & \mathrm{~A}_{55}
\end{array}\right]\left\{\begin{array}{l}
\varepsilon_{4}{ }^{\circ} \\
\varepsilon_{5}^{0}
\end{array}\right\}=\left\{\begin{array}{l}
\mathrm{Q}_{1} \\
\mathrm{Q}_{2}
\end{array}\right\}
$$

The $Q_{i}$ terms represent resultant transverse shearing forces.

The derivation of the plate stiffness matrix includes the newly formed laminate constitutive matrix. Following energy principles as described by Ochoa and Reddy, ${ }^{5}$ the stiffness relation of the element is derived as follows:

$$
\left.K=\iint_{A}([D][\Psi])^{T}[A \text { B I B D }](D][\Psi]\right) d A
$$

The basic element derivation follows the Mindlin plate assumptions extended to shear deformable composite laminates. ${ }^{5,6,9}$ Therefore, five degrees of freedom are needed: the in-plane translations ( $u$ and $v)$, the transverse translation (w), and the rotations about the two in-plane axes $\left(\phi_{1}\right.$ and $\left.\phi_{2}\right)$. The straindisplacement relations are expressed in matrix form: ${ }^{5,6}$

$$
\left\{\begin{array}{l}
\varepsilon_{1}^{0} \\
\varepsilon_{2}^{0} \\
\varepsilon_{6}^{0} \\
\kappa_{1} \\
\kappa_{2} \\
\kappa_{6} \\
\varepsilon_{5}^{0} \\
\varepsilon_{4}^{0}
\end{array}\right\}=\left[\begin{array}{ccccc}
\partial / \partial x & 0 & 0 & 0 & 0 \\
0 & \partial / \partial y & 0 & 0 & 0 \\
\partial / \partial y & \partial / \partial x & 0 & 0 & 0 \\
0 & 0 & 0 & \partial / \partial x & 0 \\
0 & 0 & 0 & 0 & \partial / \partial y \\
0 & 0 & 0 & \partial / \partial y & \partial / \partial x \\
0 & 0 & \partial / \partial x & 1 & 0 \\
0 & 0 & \partial / \partial y & 0 & 1
\end{array}\right]\left\{\begin{array}{l}
u \\
v \\
w \\
\phi_{1} \\
\phi_{2}
\end{array}\right\}
$$

However, to be successful in determining the stiffness for non-rectangular elements, an isoparametric mapping to the double-unit square is used:

$$
\begin{array}{r}
\left.\mathrm{K}=\int_{-1}^{+1} \int_{-1}^{+1}(\mathrm{D}(\xi, \eta)][\Psi(\xi, \eta)]\right)^{\mathrm{T}}[\mathrm{A} \mathrm{B} \mathrm{|} \mathrm{B} \mathrm{D}(\xi, \eta)] \\
([\mathrm{D}(\xi, \eta)][\psi(\xi, \eta)]) \\
\mathrm{J}(\xi, \eta) \mid \mathrm{d} \xi \mathrm{d} \eta
\end{array}
$$

The [A B I B D] matrix transforms by a simple rotation. The material axis $(x)$ is rotated by an angle into the new axis $(\xi)$. The shape functions are developed for the double-unit square leaving only the strain-displacement relations to be converted into the new form. The Jacobian of the mapping determines the changes required to map the material coordinate derivatives into the isoparametric derivatives. 
The basic plate element uses four corner nodes. Linear shape functions are used for each axis direction. The final step is integration of the assembled system of equations. Gaussian integration suggests using four gauss points to most accurately integrate. However, it has been determined that shear locking is very likely for such elements. A fairly reliable way to avoid shear locking is to use reducod integration. ${ }^{10-12}$ One gauss integration point is thus used. This reduces the accuracy of the integration, but the overall accuracy is acceptable with a sufficient mesh. The integration of the plate relations (omitting transverse shear) creates a $5 \mathrm{~N} \times 5 \mathrm{~N}$ stiffness matrix where $\mathrm{N}$ is the number of nodes per element. To get a sense of stiffness contribution, each stiffness matrix is separated into $5 \times 5$ stiffness sub-matrices for each nodal combination $(i, j)$ :

$$
\mathrm{K}_{\text {basic plate }}^{\mathrm{ij}}=\left[\begin{array}{ccccc}
\mathrm{K}_{11} & \mathrm{~K}_{12} & 0 & \mathrm{~K}_{14} & \mathrm{~K}_{15} \\
\mathrm{~K}_{21} & \mathrm{~K}_{22} & 0 & \mathrm{~K}_{24} & \mathrm{~K}_{25} \\
0 & 0 & 0 & 0 & 0 \\
\mathrm{~K}_{41} & \mathrm{~K}_{42} & 0 & \mathrm{~K}_{44} & \mathrm{~K}_{45} \\
\mathrm{~K}_{51} & \mathrm{~K}_{52} & 0 & \mathrm{~K}_{54} & \mathrm{~K}_{55}
\end{array}\right]
$$

Integration of the stiffness formulation with the transverse shear relations produces the following $5 \times 5$ sub-matrix for each nodal combination $(i, j)$ :

$$
\overline{\mathrm{K}}_{\text {transv. shear }}^{\mathrm{ij}}=\left[\begin{array}{ccccc}
0 & 0 & 0 & 0 & 0 \\
0 & 0 & 0 & 0 & 0 \\
0 & 0 & \overline{\mathrm{K}}_{33} & \overline{\mathrm{K}}_{34} & \overline{\mathrm{K}}_{35} \\
0 & 0 & \overline{\mathrm{K}}_{43} & \overline{\mathrm{K}}_{44} & \overline{\mathrm{K}}_{45} \\
0 & 0 & \overline{\mathrm{K}}_{53} & \overline{\mathrm{K}}_{54} & \overline{\mathrm{K}}_{55}
\end{array}\right]
$$

Combining the basic plate and transverse shear stiffness relations result in a comprehensive plate stiffness. This leads to the desined stiffness formulation for the four-noded plate element. The consistent mass is computed by integrating the density distribution through the thickness:

$$
\left\{\begin{array}{l}
\mathbf{M}_{0} \\
\mathbf{M}_{1} \\
\mathbf{M}_{2}
\end{array}\right\}=\sum_{k=1}^{\mathbf{N}} \int_{\mathbf{z}_{k}}^{\mathbf{z}_{k+1}} \rho^{(k)}\left\{\begin{array}{l}
1 \\
\mathbf{z} \\
\mathbf{z}^{2}
\end{array}\right\} d z
$$

The result is the $5 \times 5$ consistent mass sub-matrix for each nodal combination $(i, j)$ :

$$
\mathbf{M}_{\text {consistent }}^{\mathrm{ij}}=\left[\begin{array}{ccccc}
\mathbf{M}_{0} & 0 & 0 & \mathbf{M}_{1} & 0 \\
0 & \mathbf{M}_{0} & 0 & 0 & \mathbf{M}_{1} \\
0 & 0 & \mathbf{M}_{0} & 0 & 0 \\
\mathbf{M}_{1} & 0 & 0 & \mathbf{M}_{2} & 0 \\
0 & \mathbf{M}_{1} & 0 & 0 & \mathbf{M}_{2}
\end{array}\right]
$$

The $\mathbf{M}_{1}$ terms are the rotatory inertia terms and are omitted due to their small magnitude compared to $\mathrm{M}_{0}$ and $\mathbf{M}_{2}$.

This procedure is then extended to a shallow shell formulation using curvature relations: ${ }^{13,14}$

$$
\begin{aligned}
& \mathbf{k}_{1}=-\frac{\partial^{2} z}{\partial x^{2}} \\
& \mathbf{k}_{2}=-\frac{\partial^{2} z}{\partial y^{2}} \\
& \mathbf{k}_{6}=-\frac{\partial^{2} z}{\partial x \partial y}
\end{aligned}
$$

The strain-displacement relation thus becomes:

$$
\left\{\begin{array}{l}
\varepsilon_{1}^{0} \\
\varepsilon_{2}^{0} \\
\varepsilon_{6}^{0} \\
\kappa_{1} \\
\kappa_{2} \\
\kappa_{6} \\
\varepsilon_{5}^{0} \\
\varepsilon_{4}^{0}
\end{array}\right\}=\left[\begin{array}{ccccc}
\partial / \partial x & 0 & k_{1} & 0 & 0 \\
0 & \partial / \partial & k_{2} & 0 & 0 \\
\partial / \partial y & \partial / \partial x & 2 k_{6} & 0 & 0 \\
0 & 0 & 0 & \partial / \partial x & 0 \\
0 & 0 & 0 & 0 & \partial / \partial y \\
0 & 0 & 0 & \partial / \partial y & \partial / \partial x \\
0 & 0 & \partial / \partial x & 1 & 0 \\
0 & 0 & \partial / \partial y & 0 & 1
\end{array}\right]\left\{\begin{array}{l}
u \\
v \\
w \\
\phi_{1} \\
\phi_{2}
\end{array}\right\}
$$

Integration of the stiffness formulation with the shallow shell curvature relations produces the following additional $5 \times 5$ sub-matrix for each nodal combination $(\mathrm{i}, \mathrm{j})$ :

$$
\overline{\mathrm{K}}_{\text {curvature }}^{\mathrm{ij}}=\left[\begin{array}{ccccc}
0 & 0 & \overline{\mathrm{K}}_{13} & 0 & 0 \\
0 & 0 & \overline{\mathrm{K}}_{23} & 0 & 0 \\
\overline{\mathrm{K}}_{31} & \overline{\mathrm{K}}_{32} & \overline{\mathrm{K}}_{33} & \overline{\mathrm{K}}_{34} & \overline{\mathrm{K}}_{35} \\
0 & 0 & \overline{\mathrm{K}}_{43} & 0 & 0 \\
0 & 0 & \overline{\mathrm{K}}_{53} & 0 & 0
\end{array}\right]
$$

Eight nodes are used for the shallow laminated composite shell element. The total stiffness formulation for each nodal combination is:

$$
\mathbf{K}^{\mathrm{ij}}=\mathrm{K}_{\text {besic place }}^{\mathrm{ij}}+\overline{\mathrm{K}}_{\text {transv. shear }}^{\mathrm{ij}}+\overline{\mathrm{K}}_{\text {curvature }}^{\mathrm{ij}}
$$


A numerical verification of the above mentioned four- and eight-noded elements has been computed. This compares the natural frequencies of a fibrous composite laminate with an analytical solution.

\section{3-D Formulation}

From the elastic porous material theory developed by Bolton, et al. ${ }^{2}$ the stress-strain relationships between fluid and solid strains and stresses can be derived by writing the equilibrium equations for the forces acting on a unit volume of elastic porous material.

$$
\begin{gathered}
\sigma_{x}=2 \mathrm{Ne}_{\mathrm{x}}+\mathrm{Ae}_{\mathrm{z}}+\mathrm{Q} \varepsilon \\
\sigma_{\mathrm{y}}=2 \mathrm{Ne}_{\mathrm{y}}+\mathrm{Ae}_{\mathrm{z}}+\mathrm{Q} \varepsilon \\
\sigma_{\mathrm{z}}=2 \mathrm{Ne} \mathrm{e}_{\mathrm{z}}+\mathrm{Ae} \mathrm{e}+\mathrm{Q} \varepsilon \\
\mathrm{s}=\mathrm{R} \varepsilon+\mathrm{Qe} \mathrm{e}_{\mathrm{s}} \\
\tau_{\mathrm{xy}}=\tau_{\mathrm{yx}}=\mathrm{N} \gamma_{\mathrm{xy}} \\
\tau_{\mathrm{xz}}=\tau_{\mathrm{xx}}=\mathrm{N} \gamma_{\mathrm{xz}} \\
\tau_{\mathrm{yz}}=\tau_{\mathrm{zy}}=\mathrm{N} \gamma_{\mathrm{yz}}
\end{gathered}
$$

where $e_{s}=$ solid volumetric strain, $\varepsilon=$ fluid volumetric, $\mathbf{N}=$ elastic shear modulus, $A=$ first Lame constant, $Q=(1-h) * E_{2}$ and $R=h * E_{2}$, where $h=$ porosity, $E_{2}=$ bulk modulus of elasticity of the fluid in the porous medium.

The differential equations of equilibrium for the solid phase of the elastic porous material result in:

$$
\begin{aligned}
& \frac{\partial \sigma_{\mathrm{z}}}{\partial \mathrm{x}}+\frac{\partial \tau_{\mathrm{yx}}}{\partial \mathrm{y}}+\frac{\partial \tau_{\mathrm{x}}}{\partial \mathrm{z}}= \\
& \rho_{1} \frac{\partial^{2} \mathrm{u}_{\mathrm{x}}}{\partial \mathrm{t}^{2}}+\rho_{2}\left(\mathrm{q}^{2}-1\right) \frac{\partial^{2}}{\partial \mathrm{t}^{2}}\left(\mathrm{u}_{\mathrm{x}}-\mathrm{U}_{\mathrm{z}}\right)+b \frac{\partial}{\partial t}\left(\mathrm{u}_{\mathrm{z}}-\mathrm{U}_{\mathrm{x}}\right) \\
& \frac{\partial \tau_{\mathrm{xy}}}{\partial \mathrm{x}}+\frac{\partial \sigma_{y}}{\partial \mathrm{y}}+\frac{\partial \tau_{\mathrm{zy}}}{\partial \mathrm{z}}= \\
& \rho_{1} \frac{\partial^{2} \mathrm{u}_{\mathrm{y}}}{\partial^{2}}+\rho_{2}\left(\mathrm{q}^{2}-1\right) \frac{\partial^{2}}{\partial \mathrm{t}^{2}}\left(\mathrm{u}_{\mathrm{y}}-\mathrm{U}_{\mathrm{y}}\right)+b \frac{\partial}{\partial \mathrm{t}}\left(\mathrm{u}_{\mathrm{y}}-\mathrm{U}_{\mathrm{y}}\right) \\
& \frac{\partial \tau_{\mathrm{xz}}}{\partial \mathrm{x}}+\frac{\partial \tau_{\mathrm{yz}}}{\partial \mathrm{y}}+\frac{\partial \sigma_{\mathrm{z}}}{\partial \mathrm{z}}= \\
& \rho_{1} \frac{\partial^{2} \mathrm{u}_{\mathrm{z}}}{\partial^{2}}+\rho_{2}\left(\mathrm{q}^{2}-1\right) \frac{\partial^{2}}{\partial^{2}}\left(\mathrm{u}_{\mathrm{z}}-\mathrm{U}_{\mathrm{z}}\right)+b \frac{\partial}{\partial t}\left(\mathrm{u}_{\mathrm{z}}-\mathrm{U}_{\mathrm{z}}\right)
\end{aligned}
$$

where $q^{2}=$ structure factor (approaches unity if pores are straight and uniform), $\rho_{1}=$ bulk density of solid phase, $\rho_{2}=$ bulk density of the fluid phase, $b=$ viscous coupling factor associated to flow resistivity. The left term represents the net force per unit volume.
From the three right terms, the first one represents the acceleration of the solid, the second the momentum transfer between solid and fluid from the pore tortuosity, and the third the viscous coupling forces associated with the flow resistivity. Similar differential equations can be derived for the fluid phase:

$$
\begin{aligned}
& \frac{\partial s}{\partial \mathrm{x}}=\rho_{2} \frac{\partial^{2} \mathrm{U}_{\mathrm{x}}}{\partial \mathrm{t}^{2}}+\rho_{2}\left(\mathrm{q}^{2}-1\right) \frac{\partial^{2}}{\partial \mathrm{t}^{2}}\left(\mathrm{U}_{\mathrm{x}}-\mathrm{u}_{\mathrm{x}}\right)+b \frac{\partial}{\partial t}\left(\mathrm{U}_{\mathrm{x}}-\mathrm{u}_{\mathrm{x}}\right) \\
& \frac{\partial s}{\partial \mathrm{y}}=\rho_{2} \frac{\partial^{2} \mathrm{U}_{\mathrm{y}}}{\partial \mathrm{t}^{2}}+\rho_{2}\left(\mathrm{q}^{2}-1\right) \frac{\partial^{2}}{\partial \mathrm{t}^{2}}\left(\mathrm{U}_{\mathrm{y}}-\mathrm{u}_{\mathrm{y}}\right)+b \frac{\partial}{\partial t}\left(\mathrm{U}_{\mathrm{y}}-\mathrm{u}_{\mathrm{y}}\right) \\
& \frac{\partial s}{\partial \mathrm{z}}=\rho_{2} \frac{\partial^{2} \mathrm{U}_{\mathrm{z}}}{\partial \mathrm{t}^{2}}+\rho_{2}\left(\mathrm{q}^{2}-1\right) \frac{\partial^{2}}{\partial \mathrm{t}^{2}}\left(\mathrm{U}_{\mathrm{z}}-\mathrm{u}_{\mathrm{z}}\right)+b \frac{\partial}{\partial t}\left(\mathrm{U}_{\mathrm{z}}-\mathrm{u}_{\mathrm{z}}\right)
\end{aligned}
$$

The following steps can be followed to derive the system of equations in matrix form:

\section{Assume a harmonic time dependency of motion.}

2. Develop the weak form of the differential equations by multiplying each one by a weighting function and integrating over the volume of an element.

3. Express the stress in terms of displacements using the stress strain relations, and use the Green's theorem to lower the order of differentiation on the displacement variables.

4. Utilize the weighted residual method to derive the three dimensional finite element for a foam element:

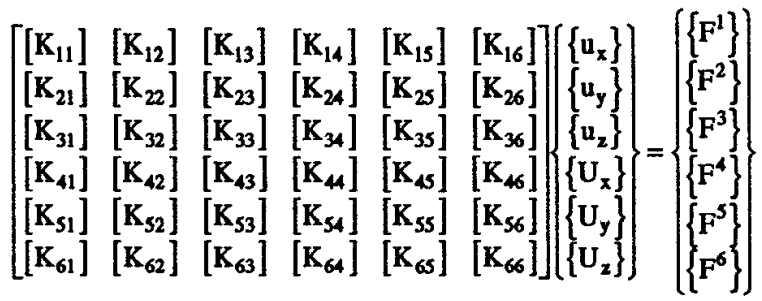

The global system of equations for the elastic porous material can be obtained by utilizing continuity of primary variables (displacement components of the elastic porous body) and the balance of secondary variables at connecting nodes (normal and shear stresses acting on the solid and shear face).

The coupling between the beam approximation of the structural panel and the two-dimensional elastic porous finite elements is the model by which the composite shell elements are joined with the threedimensional elastic porous brick elements. In such a way, a general sound absorption system using 
composite laminates and elastic porous materials is successfully modeled.

\section{Conclusions}

The investigation into the two-dimensional form of the elastic porous finite element indicates the coupling inherent along the interface between the foam and the structural panel. The vibrational equations of motion are determined for a composite laminate with shear deformation. In three dimensions, the composite laminate formulation of vibrations combines with the development of a brick type elastic porous finite element. In such a way, the acoustic vibration of the system is modeled to give an indication of transmission loss. Future work includes verifying the numerical solutions with experimental data and implementing the formulations with an existing general purpose acoustical finite element analysis code, COMET/Acoustics. ${ }^{15}$

The development of the finite element formulation for elastic porous materials has opened up an area of designing low weight sound absorption systems with maximum transmission loss. The expected growth of the use of composite laminates in transportation applications requires the ability to reliably predict the response of these systems. The three-dimensional finite element method for a combined composite laminate and elastic porous panel is this valuable tool for the sound absorption systems of the future.

\section{References}

1. Allard, J.F., Propagation of Sound in Porous Media: Modelling Sound Absorbing Materials, Elsevier Applied Science, New York, 1993, pp. 96105.

2. Bolton, J.S., N.-M. Shiau and Y.J. Kang, "Sound Transmission Through Multi-Panel Structures Lined with Elastic Porous Materials," J. Sound Vib., In Press, 1995.

3. Biot, M.A., "Theory of Propagation of Elastic Waves in Fluid-Saturated Porous Solid. I. Low frequency range. II. High frequency range," J. Acoust. Soc. Am., Vol. 28, 1956, pp. 168-191.

4. Kang, Y.J, J.S. Bolton, W. Tsoi and C. Mollo, "Acoustical Finite Element Model of Elastic Porous Materials", Presented at ASME Conference, Troy, MI, April 5-7, 1995.
5. Ochoa, O.O., and Reddy, J.N., Finite Element Analysis of Composite Laminates, Kluwer Academic Publishers, Boston, 1992, pp. 37-109.

6. Noor, A.K. and Mathers, M.D., "Shear-Flexible Finite-Element Models of Laminated Composite Plates and Shells", NASA Technical Note, D-8044, Dec. 1975.

7. Anderson, William J., Finite Elements in Mechanical and Structural Design: Dynamic and Nonlinear Analysis, Automated Analysis Corp., Ann Arbor, MI, May 1985.

8. Whitney, James M., Structural Analysis of Laminated Anisotropic Plates, Technomic Publishing Co., Inc., Lancaster, PA, 1987.

9. Koo, K.N. and Lee, I., "Vibration and Damping Analysis of Composite Laminates Using Shear Deformable Finite Element," AIAA Journal, Vol. 31, No. 4, Dec. 1993, pp. 728-735.

10. Malkus, D.S. and Hughes, T.J.R., "Mixed Finite Element Methods -- Reduced and Selective Integration Techniques: A Unification of Concepts," Comp. Meth. App. Mech. Engng., Vol. 15 (1978), pp. 63-81.

11. Pugh, E.D.L., Hinton, E., and Zienkiewicz, O.C., "A Study of Quadrilateral Plate Bending Elements with 'Reduced' Integration," Int. J. Num. Meth. Engng., Vol. 12 (1978), pp.1059-1079.

12. Zienkiewicz, O.C., Taylor, R.L, and Too, J.M., "Reduced Integration Technique in General Analysis of Plates and Shells," Int. J. Num. Meth. Engng., Vol. 3 (1971), pp. 275-290.

13. Roa, K.P., "A Rectangular Laminated Anisotropic Shallow Thin Shell Finite Element," Comp. Meth. App. Mech. Engng., Vol. 15 (1978), pp. 13-33.

14. Flugge, Wilhelm, Stresses in Shells, SpringerVerlag, New York, 1990, pp. 414-422.

15. COMET/Acoustics User Document, "Chapter 7: Theory," Automated Analysis Corp., Ann Arbor, MI, July 1994. 


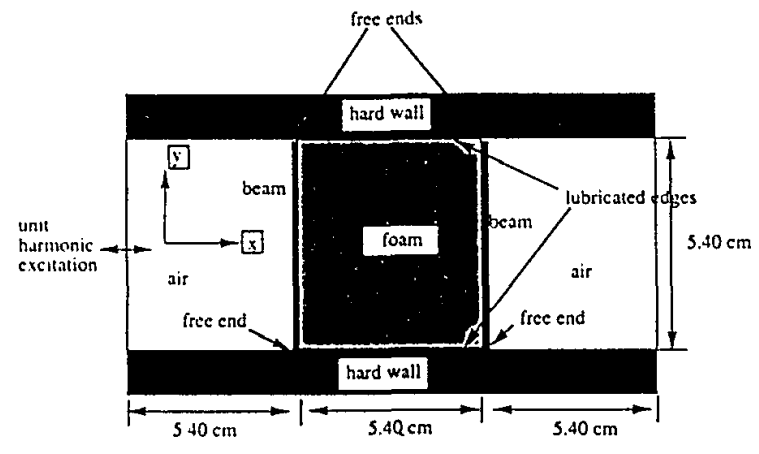

Figure 1: Geometry of plates bonded to sandwiched foam layer

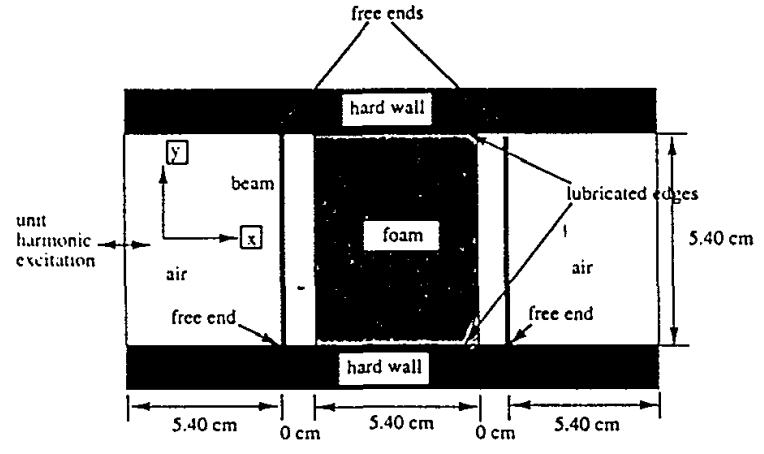

Figure 2: Geometry of plates not bonded to sandwiched foam layer

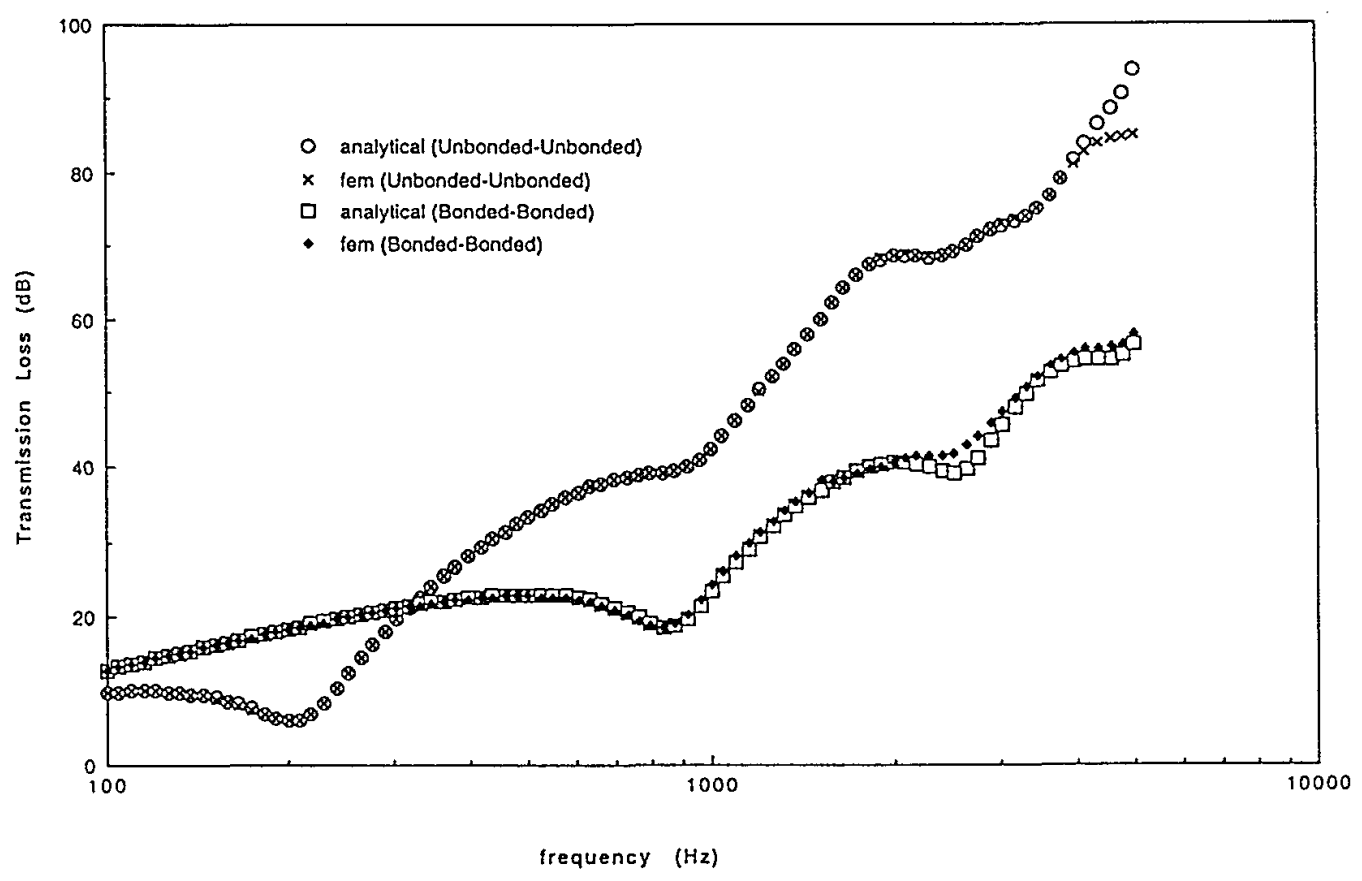

Figure 3: Comparison of frequency dependent transmission loss calculation for both finite element and analytical methods for both bonded and not bonded geometries. 